\title{
Mikhail Lomonossov, le premier scientifique russe moderne
}

Cet article, consacré à Mikhail Lomonossov (1711-1765), avait pour moi plusieurs justifications : un intérêt pour la chimie physique autour des travaux du savant russe Venjamin Levich (1917-1987), auteur de l'ouvrage de référence Physicochemical Hydrodynamics [1] et héritier du concept de " chimie physique » introduit par Lomonossov ; une visite du Kuntskamera, le premier musée de science du monde, créé par Pierre le Grand à Saint-Pétersbourg, dont toute une partie est consacrée à l'œuvre de ce savant polymathe du siècle des Lumières... et la lecture de deux articles de Vladimir Shiltsev, écrits en 2011 à l'occasion du tricentenaire de la naissance de Lomonossov. Nous avons traduit et adapté le texte publié dans APS News [2], en utilisant des éléments de I'article plus complet de Physics Today [3].

Étienne Guyon

PMMH, ESPCI, Paris

\section{Références}

1 V. G. Levich, Physicochemical Hydrodynamics, Prentice Hall (1962).

2• V. Shiltsev, “Nov. 19, 1711: Birth of Mikhail Lomonosov, Russia's first modern scientist“, This Month in Physics History, APS News, 20, n'10 (novembre 2011).

3• V. Shiltsev, "Mikhail Lomonosov and the dawn of Russian science", Physics Today 65 (2012) 40-46.

4• M.V. Lomonosov, Complete Works, 11 vols., S. Vavilov, S. Kravets, eds., Akad. Nauk. SSSR, Moscou et Leningrad (1950-83).
Mikhail Vassilievitch Lomonossov est né le 19 novembre $1711^{\text {(a) }}$ dans un village de la Russie du nord près d'Archangelsk, au sein d'une famille de serfs de la Couronne devenus pêcheurs. À l'âge de 19 ans, il s'échappe de chez lui avec ses deux livres favoris (Grammatica et Arithmetica) pour faire des sciences.

Un voyage de cinq semaines et 1300 kilomètres de route enneigée dans un convoi de luges qui transportait du poisson gelé, le conduit à Moscou où, après avoir menti en disant qu'il était fils d'un noble, il fut admis à l'académie slave-grecque-latine du monastère Spassky. Mourant à moitié de faim avec trois kopeks par jour, il mena à bien en quatre ans un programme de huit ans en latin, grec, slavon, géographie, histoire, philosophie et catéchisme. De là, il fut admis à l'Académie des Sciences de Saint-Pétersbourg pour continuer ses études parmi les douze meilleurs élèves de l'année 1736.

L'Académie envoya alors Mikhail à l'Université de Marburg (Allemagne) où il étudia les mathématiques, la chimie, la physique, l'hydraulique, les mines, les humanités, avec Christian Wolff, un savant et philosophe encyclopédiste disciple de Leibnitz, qui prit conscience des exceptionnelles possibilités scientifiques de Lomonossov.

Après avoir passé l'an 1739 à Freiburg à étudier les problèmes de mines avec Johann Heckel, Lomonossov put retourner avec quelques difficultés en Russie deux ans plus tard. Ses mérites, reconnus grâce aux rapports d'études qui étaient envoyés régulièrement de l'étranger (ainsi qu'une ode poétique adressée à l'Impératrice Anna), lui donnèrent accès à un poste d'assistant de physique à l'Académie de Saint-Pétersbourg. Ce fut le premier académicien né en Russie élu en 1745 ; il servit comme membre de la Chancellerie de l'Académie, avec la charge de toutes les activités scientifiques et d'éducation ainsi que des départements, de 1757 jusqu'à sa mort en 1765.

L'Académie des Sciences de SaintPétersbourg avait été fondée en 1724 par un décret de Pierre le Grand, à la suggestion de Leibnitz. Pilotée à sa création par des membres étrangers, l'Académie avait connu un bon départ, attirant des scientifiques notables tels que Daniel Bernoulli (17001782) et Leonhard Euler (1707-1783).

Mais, au retour de Lomonossov en Russie, ces membres prestigieux étaient partis et l'Académie était en crise, suite à une mauvaise gouvernance et à une gestion des crédits défectueuse. Lomonossov combattit énergiquement ces défauts, afin de suivre de nouveau la trace du projet de Pierre le Grand. Pour cela, il augmenta le nombre de publications en russe (en plus du latin et de l'allemand), insistant aussi pour que les académiciens donnent des enseignements réguliers en russe. Ceci conduisit à augmenter le nombre d'académiciens russes et d'étudiants du Gymnasium de l'Académie. Il créa en 1755 la première université à Moscou, qui porte aujourd'hui son nom.

La nature polymathique de ce Titan russe de l'âge des Lumières est largement 


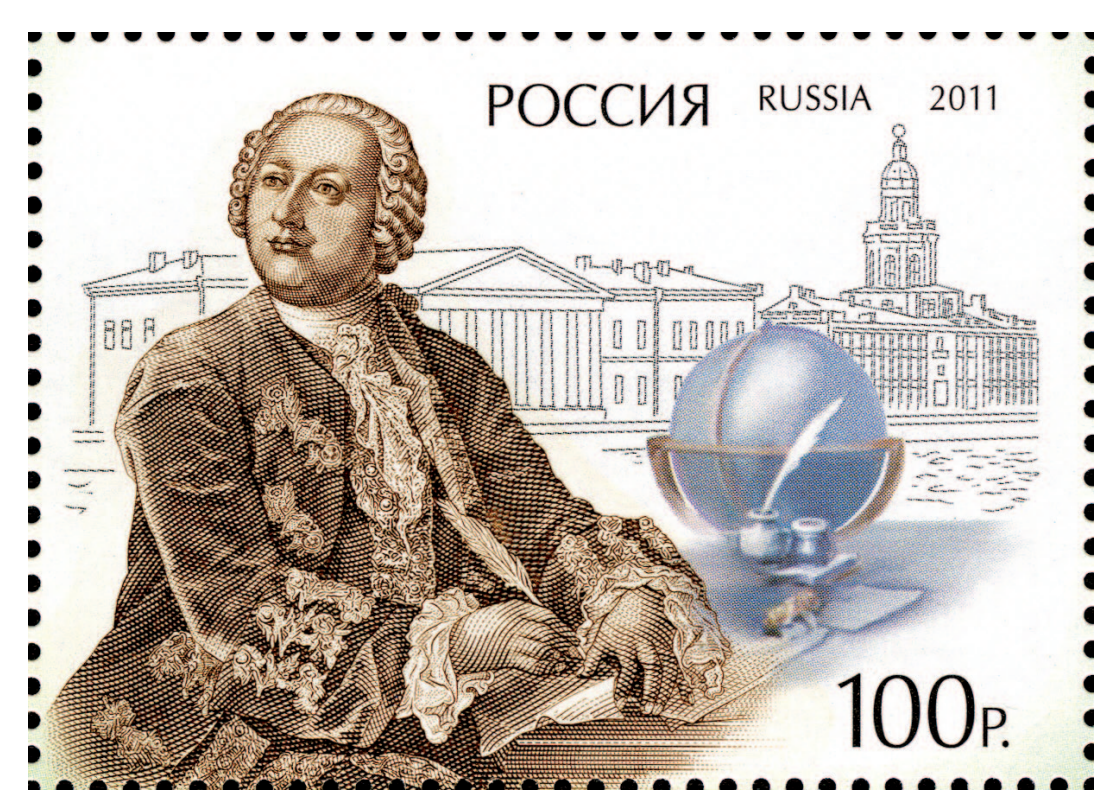

Timbre édité par la Fédération de Russie en 2011, pour le tricentenaire de la naissance de M.V. Lomonossov.

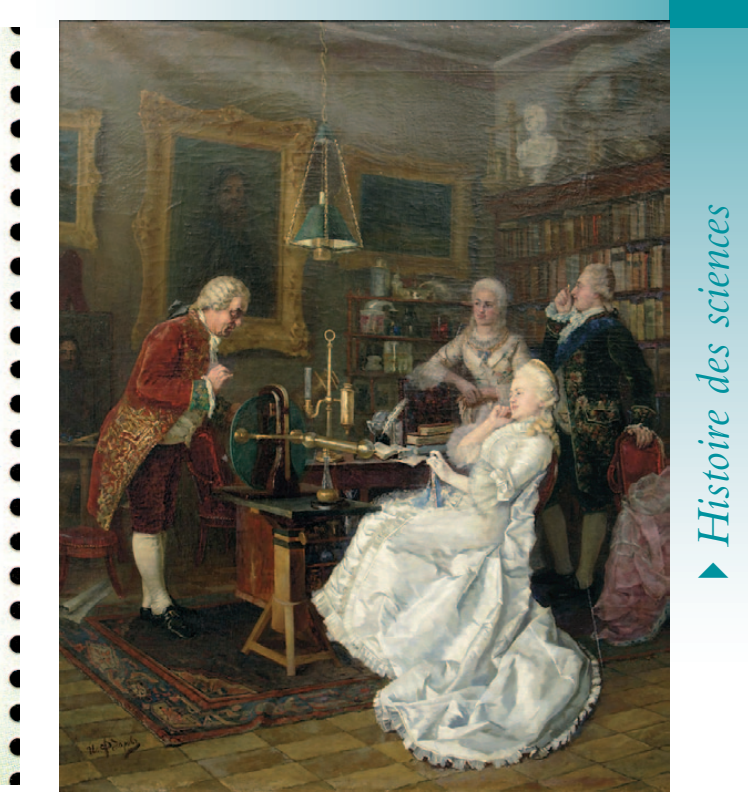

L'Impératrice Catherine II de Russie chez Lomonossov en 1764 (œuvre d'Ivan Kuzmich Fedorov, 1884). démontrée par le contenu de ses œuvres complètes [4] : vols. 1-4, physique, chimie et astronomie ; vol. 5, minéralogie, métallurgie et géologie ; vols. 7-8, philologie, poésie et prose ; vols. 9-11, correspondance, lettres et transactions.

La profondeur de sa créativité scientifique est encore plus remarquable. Dans le simple domaine des sciences naturelles ${ }^{(b)}$, Lomonossov fit à lui seul plus de 4000 tests chimiques dans le premier laboratoire national russe. Ses explications scientifiques dans tous les phénomènes physiques et chimiques se font en termes d'interactions mécaniques de particules "petites et insensibles » - nos molécules - à l'intérieur d'un éther continu. Il se représente le froid absolu comme une condition dans laquelle les corpuscules cessent leur mouvement linéaire et rotationnel ${ }^{(\mathrm{c})}$. Il créé ainsi le terme « chimie physique » en 1752.

Dix-sept ans avant Lavoisier, Lomonossov démontre la loi de conservation de la matière $^{(\mathrm{d})}$, en montrant que des plaques de plomb placées dans un récipient scellé sans accès à l'air extérieur ne changent pas de poids une fois chauffées (1756). Il fait des expériences sur l'électricité (suffisamment dangereuses pour que son collègue Georg Richmann y perde la vie lors de la formation d'une boule de feu alors que lui survécut " miraculeusement »), et il propose une théorie de l'électricité dans l'atmosphère, qui va au-delà des travaux de Franklin et rend compte des éclairs(e) et des aurores boréales. À la recherche d'un dispositif pour envoyer dans l'atmosphère des instruments météorologiques et de l'équipement électrique, il construit le premier modèle d'hélicoptère (1754) : pour assurer une compensation des couples, celui-ci comportait deux hélices tournant dans des directions opposées, actionnées par un dispositif d'horlogerie, et a pu se soulever légèrement du sol.

Pendant le transit de Vénus du 26 mai 1761, Lomonossov découvre l'existence de son atmosphère en observant l'auréole brillante au début et à la fin du transit ${ }^{(\mathrm{f})}$, et il donne une explication optique détaillée en termes de diffraction. Trente ans avant Herschel, en 1762, il invente et construit un télescope d'un type nouveau, utilisant un miroir primaire incliné de quatre degrés de façon à former une image directement sur un oculaire placé sur le côté ; plus tard, dans la même année, il invente un sidérostat qui lui a permis de suivre les étoiles en faisant tourner un miroir plan placé devant le télescope de 40 pieds, plutôt que l'ensemble du télescope.

Mikhail Lomonossov est mort le 15 avril

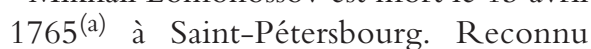
seulement au $19^{\mathrm{e}}$ siècle comme le premier personnage de l'histoire des sciences en Russie, cet homme très grand et fort (au propre et au figuré) fait penser à son idole : Pierre le Grand. Mais il n'a pas eu à l'Ouest la même reconnaissance que les savants contemporains ${ }^{(\mathrm{g})}$, en particulier parce que ses très nombreux travaux en grammaire, art de la mosaïque et poésie ${ }^{(h)}$ ont quelque peu occulté ses travaux scientifiques. Cette méconnaissance vient aussi de la brièveté de sa vie (53 ans) et du fait que sa communauté nationale resta faible jusqu'à la fin des années 1800, avec peu de contacts avec l'Ouest (si l'on excepte Euler). Le tricentenaire de sa naissance fut célébré avec éclat en Russie.

Vladimir D. Shiltsev

Directeur du Centre de physique des accélérateurs au Fermi Lab (Batavia, Illinois, USA) Auteur invité d'APS News

(a) Dates du calendrier grégorien ; soit 8 novembre 1711 et 4 avril 1765 dans notre calendrier julien.

(b) Il semble que Lomonossov n'ait jamais fait appel aux équations dans ses travaux publiés!

(c) À l'aide d'un mélange de glace et d'acide nitrique il obtient une température de $-38^{\circ} \mathrm{C}$, qui lui permet d'étudier les propriétés du mercure à l'état solide.

(d) "Tous les changements dans la nature se font... de façon que quelle que soit la matière ajoutée au corps, la même quantité doit être enlevée à un autre. Comme c'est une loi générale de la nature, elle s'applique aux lois du mouvement : un corps perd autant de mouvement qu'il en communique à un autre. " Cette approche cartésienne contraste avec la vision newtonienne privilégiée à l'époque !

(e) Lomonossov se représente l'éclair comme dû à la friction entre les couches d'air chaud (ascendantes) et l'air froid descendant dans l'atmosphère, l'électricité étant accumulée sur des microparticules " huileuses ".

(f) La paternité de cette découverte ne lui fut reconnue qu'au milieu du $20^{\mathrm{e}}$ siècle. Lomonossov alla jusqu'à émettre l'hypothèse de la possibilité d'une vie sur Vénus !

(g) En particulier si on compare sa carrière à celle, analogue, de Benjamin Franklin qui fut son contemporain et aussi une référence de ce siècle des Lumières, et qui contribua comme Lomonossov à faire entrer leurs pays respectifs dans l'ère moderne.

(h) «La poésie est ma consolation, la physique ma profession." 